\title{
Tuning Polyelectrolyte-Surfactant Interactions: Modification of Poly(ethylenimine) with Propylene Oxide and Blocks of Ethylene Oxide
}

\author{
J. Penfold, ${ }^{*}{ }^{\dagger}{ }^{\ddagger}$ R. K. Thomas, ${ }^{\ddagger}$ P. Li, ${ }^{\dagger}$ S. N. Batchelor, ${ }^{\S}$ I. M. Tucker, ${ }^{\S}$ and A. W. Burley ${ }^{\S}$ \\ ${ }^{\dagger}$ ISIS, STFC, Rutherford Appleton Laboratory, Chilton, Didcot, OXON OX1 OQX, United Kingdom \\ ${ }^{\ddagger}$ Physical and Theoretical Chemistry Laboratory, Oxford University, South Parks Road, Oxford OX1 3QZ, United Kingdom \\ ${ }^{\S}$ Unilever Research and Development Laboratory, Port Sunlight, Quarry Road East, Bebington, Wirral CH62 4ZD, United Kingdom
}

Supporting Information

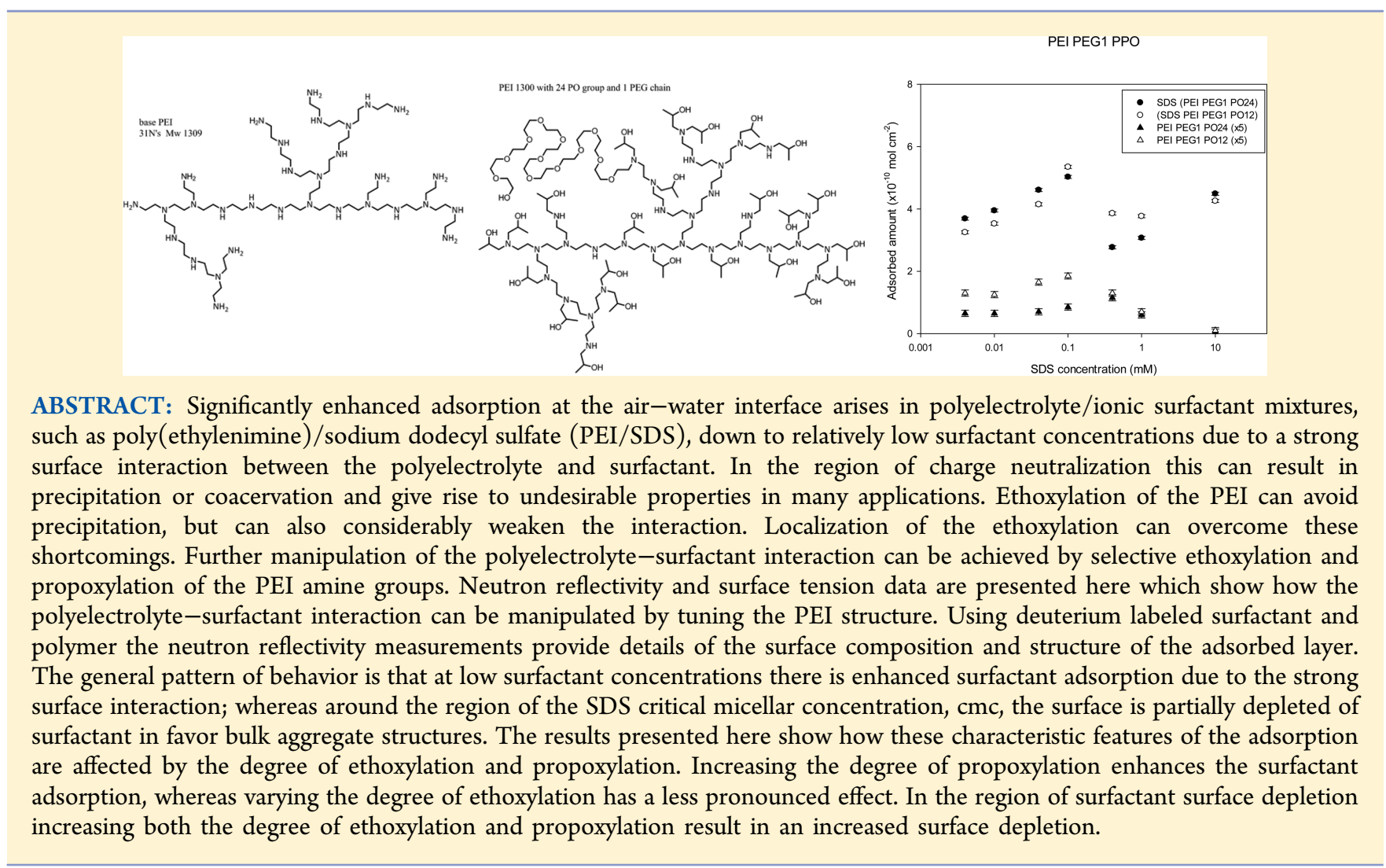

\section{INTRODUCTION}

The study of polymer-surfactant interactions and their implications for self-assembly and adsorption at a variety of different interfaces has been an active area of research for a number of years. ${ }^{1-6}$ This is in part due to their extensive range of applications in detergency, cosmetics, foods, paints, drug delivery, and coatings. ${ }^{3}$ In many of the applications the polymer is used as a viscosity modifier or to manipulate self-assembly. However, it is also used extensively to modify adsorption or deposition. This has stimulated great interest in the surface adsorption properties of polymer-surfactant mixtures, ${ }^{4,5}$ and this is the focus of the paper and study.

The surface adsorption properties of weakly interacting polymer-surfactant mixtures, such as poly(ethylene oxide),
PEO/SDS, is relatively well understood. ${ }^{1,7}$ In such cases they relate directly to the variations in the solution phase behavior, and in the case of PEO/SDS is also associated with the competitive adsorption of the PEO and SDS. For the more strongly interacting polyelectrolyte/ionic surfactant mixtures the adsorption behavior is more complex. ${ }^{5,7-9}$ The strong surface interaction often results in enhanced adsorption at relatively low surfactant concentrations due to surface polymer/ surfactant complex formation, and the competition between surface and solution complex formation determines the

Received: December 2, 2015

Revised: January 11, 2016

Published: January 12, 2016 
evolution in the adsorption with surfactant concentration. ${ }^{7}$ At surfactant concentrations close to charge neutralization the solutions are often cloudy and precipitation/coacervation can occur. Depending upon the nature of the concentrated precipitated or coacervated phase in general two broadly different types of surface behavior occur. For systems such as SDS/poly(dimethyldiallyl ammonium chloride), polydmdaac, ${ }^{10}$ where nondissociated solution complexes are formed in the precipitation/coacervation region partial depletion from the surface occurs and this coincides with a peak in the surface tension. Where partially dissociated solution complexes are formed, for example poly(styrenesulfonate), PSS/dodecyltrimetyl ammonium bromide, DTAB, ${ }^{11}$ an extended plateau region in the surface tension exists and the surface structure is more complex, consisting of ordered layered structures from a trilayer to multiple bilayers. The surface multilayer formation is due to a wetting of the surface by the concentrated precipitate/ coacervate phase which has a lower surface tension and hence is more surface active than the dilute phase that is in equilibrium with it. These general features are described thermodynamically by recent theoretical developments.?

Precipitation in ionic surfactants is a commonplace phenomena and can be induced as described above by polyelectrolytes, or more commonly by the addition of multivalent counterions such as $\mathrm{Ca}^{2+}$ and $\mathrm{Al}^{3+}$. For example, the addition of multivalent counterions to solutions of SDS or other related anionic surfactants leads rapidly to precipitation. $^{12-16}$ This results in a reduction in the properties of surfactant solutions in applications such as detergency, the familiar action of hard water, but can be advantageous in water and solid contaminant remediation. ${ }^{13,14,16}$ To minimize the negative impacts a range of different strategies to improve solubility have been developed, and include the addition of sequesters, or cosurfactants and modification of the anionic surfactant structure. ${ }^{13,17,18}$ In this context polyelectrolytes can be considered as polycations or polyanions and precipitation can be also an important phenomena in polyelectrolyte/ionic surfactant mixtures.

Polyethylenimine, PEI, is a particularly important polyelectrolyte, and it exists with both linear and branched architectures. The charge on PEI is $\mathrm{pH}$ dependent ${ }^{19}$ and at low $\mathrm{pH}$ it has the highest charge density of any polyelectrolyte. Furthermore, the structure can be readily modified by ethoxylation or the addition of hydrophobic or hydrophilic units. $^{20-22}$ It has been extensively exploited in a wide variety of applications, and notably in a range of biomedical applications involving DNA compaction, DNA delivery, and different gene therapy applications. ${ }^{21-27}$ In the context of the interaction of PEI with surfactants, the adsorption and self-assembly properties of PEI and SDS mixtures have been extensively studied. ${ }^{19,29,28-31}$ Zhang et al. ${ }^{28}$ used NR to show how the adsorption of SDS was enhanced down to very low SDS concentrations due to the strong surface interaction between the SDS and PEI. For linear PEI the adsorption was in the form of a monolayer and was largely independent of $\mathrm{pH}$. For branched PEI there was a tendency to form surface layered structures at higher $\mathrm{pH}$ and at higher SDS concentrations. Ethoxylation of the PEI offers a suitable route to increase the polyelectrolyte solubility and reduce the effects of precipitation when charge neutralization is approached by the addition of oppositely charged surfactants. Penfold et al. ${ }^{32}$ showed that for relatively high degrees of ethoxylation, $\mathrm{PEI}-\mathrm{EO}_{7}$, where there is on average an $\mathrm{EO}_{7}$ chain attached to each amine group, the strong interaction between the SDS and PEI is significantly reduced and the associated advantages of enhanced adsorption lost. Indeed the behavior of SDS - PEI-EO 7 mixtures resembled that of a weakly interacting nonionic polymersurfactant mixture. ${ }^{1}$ Zhang et al. ${ }^{33}$ subsequently showed that for degrees of ethoxylation or propoxylation of less than three ethylene oxide (or propylene oxide) groups attached to each amine group the strong interaction between PEI and SDS is preserved. Batchelor et al. ${ }^{34}$ showed most recently that by the ethoxylation of PEI into discrete regions the precipitation effects upon the addition of SDS are suppressed while the favorable enhanced adsorption properties are retained.

We demonstrate in this paper how the surface properties in modified PEI-SDS mixtures can be further manipulated as a result of tuning the polyelectrolyte-surfactant interaction and behavior by the selective addition of ethylene oxide chains and propylene oxide groups.

\section{EXPERIMENTAL DETAILS}

(i). Surface Tension. The surface tension, ST, measurements were made on a Krüss K100 tensiometer by the duNouy method and using a platinum-iridium ring. The ring was rinsed in high purity water and dried in a Bunsen flame between measurements. The tensiometer was calibrated in high purity water for a value of $72 \mathrm{mN} / \mathrm{m}$. The temperature was controlled at $25 \pm 1{ }^{\circ} \mathrm{C}$. Repeated measurements were made until the variation in surface tension was $<0.2 \mathrm{mN} / \mathrm{m}$. Depending upon the solution this resulted in a total lapse time per sample of up to $2 \mathrm{~h}$, and the solutions were partially covered during that time to minimize evaporation.

(ii). Neutron Reflectivity. The neutron reflectivity, NR, measurements were made at the air-solution interface on the SURF ${ }^{35}$ and INTER $^{35}$ reflectometers at the ISIS neutron source. The reflectivity, $R(Q)$ was measured as a function of the wave vector transfer, $Q$ perpendicular to the free liquid surface $(Q=4 \pi \sin \theta / \lambda ; \theta$ is the grazing angle of incidence and $\lambda$ the neutron wavelength). The useable $Q$ range of 0.03 to $0.35 \AA^{-1}$ was covered using a $\theta$ of $2.3^{\circ}$ and a $\lambda$ range of 1 to $15 \AA$ on INTER, and a $Q$ range of 0.05 to $0.35 \AA^{-1}$ was covered on SURF using a $\theta$ of $1.5^{\circ}$ and a $\lambda$ range of 1 to $7 \AA$. The measurements were made in sealed Teflon troughs ${ }^{35,36}$ with sample volumes $\sim 25 \mathrm{~mL}$ at a temperature of $25{ }^{\circ} \mathrm{C}$. The reflectivity was calibrated on an absolute scale by reference to the direct beam intensity and the reflectivity from a pure $\mathrm{D}_{2} \mathrm{O}$ surface. Each measurement made sequentially on a seven position sample changer took $\sim 15$ to $30 \mathrm{~min}$, and some measurements were repeated to ensure equilibrium. As routinely used in studying surfactant adsorption ${ }^{37}$ and polymer/surfactant adsorption ${ }^{5}$ at the air-water interface the measurements were made using deuterium labeled adsorbates in null reflecting water, $\mathrm{NRW},\left(8 \mathrm{~mol} \% \mathrm{D}_{2} \mathrm{O} / \mathrm{H}_{2} \mathrm{O}\right.$ mixture with a scattering length density of 0.0 , and so index matched to air). In this case the resulting reflectivity arises only from the adsorbed layer and is dominated by the deuterium labeled components at the interface. In the measurements made here the surfactant and modified PEI have both been deuterium labeled (see later) and two or three separate measurements were made, for both components deuterated, or one or the other deuterated only. The data are analyzed using the simplest model consistent with the data, mostly a single layer and requiring two layers in some limited regions only. The data are fitted by a leastsquares algorithm using calculated reflectivity based on the exact optical matrix formulizm, ${ }^{38,39}$ to obtain a thickness, $d$, and scattering length density, $\rho$, per layer. For the polymer/surfactant mixture and for a single layer description, the adsorbed amount or area/molecule for each component is given by ${ }^{37}$

$$
d \rho=\frac{\sum b_{1}}{A_{1}}+\frac{\sum b_{2}}{A_{2}}
$$

where $A_{i}$ is the area/molecule of component $i$ and $b_{i}$ its sum of scattering lengths. The $\Sigma b$ values for the different components are 
summarized in Table 1 . The adsorbed amount, $\Gamma$, is then estimated from $\Gamma=1 / N_{\mathrm{a}} A$, and $N_{\mathrm{a}}$ is Avogadro's number.

\section{Table 1. $\Sigma \boldsymbol{b}$ Values for SDS and Different Polymers Used}

\begin{tabular}{lc}
\multicolumn{1}{c}{ material } & $\mathrm{s}\left(\times 10^{-3} \AA\right)$ \\
d-SDS & 2.76 \\
h-SDS & 0.152 \\
PEI-PEG1-PO12 (h-PO) & 2.09 \\
PEI-PEG1-PO12 (d-PO) & 9.59 \\
PEI-PEG1-PO24 (h-PO) & 2.49 \\
PEI-PEG1-PO24 (d-PO) & 17.48 \\
PEI-PEG3-PO12 (h-PO) & 3.12 \\
PEI-PEG3-PO12 (d-PO) & 10.62 \\
PEI-PEG3-PO24 (h-PO) & 3.52 \\
PEI-PEG3-PO24 (d-PO) & 18.51 \\
PEI-PO12 (h-PO) & 1.58 \\
PEI-PO12 (d-PO) & 9.07 \\
\hline
\end{tabular}

The NR measurements were made with the isotopic combinations of d-SDS/h-polymer, dh, d-SDS/d-polymer, dd, and h-SDS/dpolymer, hd, or with the combination dd, and hd. The reflectivity profile for each isotopic combination is modeled separately as a single monolayer to obtain the product $\mathrm{d} \times \rho$, where $\mathrm{d}$ is the layer thickness and $\rho$ the scattering length density. The simultaneous equations arising from the application of eq 1 using the $\mathrm{d} \times \rho$ values from the isotopic combinations dd, hd or $\mathrm{dh}$, hd were solved to obtain the adsorbed amounts of each component. In some limited cases all 3 contrasts, dh, $\mathrm{hd}$, and dd, were measured. However, the differences in the reflectivity for $\mathrm{dd}$ and $\mathrm{dh}$, where the SDS adsorption dominates, are sufficiently small that they are similar to the systematic errors, and using all three contrasts resulted in larger errors in the adsorbed amounts of the individual components.

(iii). Materials and Measurements Made. The deuterium labeled SDS, d-SDS $\left(\mathrm{C}_{12} \mathrm{D}_{25} \mathrm{SO}_{4} \mathrm{Na}\right)$ and hydrogeneous SDS, h-SDS $\left(\mathrm{C}_{12} \mathrm{H}_{25} \mathrm{SO}_{4} \mathrm{Na}\right)$ were obtained from the ISIS Isotope Facility ${ }^{40}$ and were synthesized and purified by recrystallization from ethanol as previously described. ${ }^{41}$ The purity of the surfactant was verified by the absence of a minimum in the surface tension at the critical micellar concentration, $\mathrm{cmc}$, and from the adsorption value at a concentration above the $\mathrm{cmc}$, as measured by NR. The modified PEI's were derived from a branched PEI with a MW of $1300 \mathrm{Da}$, as supplied by SigmaAldrich. A single or three poly(ethylene oxide), EO, chains (with 12 ethylene oxide groups) were attached as described previously, ${ }^{34}$ and subsequently 12 or 24 propylene oxide, PO groups, were attached singly to the available amine groups, as illustrated in Figure 1 for PEIPEG1-PO24

Identical polymers, but with either h-PO or d-PO groups attached were prepared. The nomenclature for the different modified PEI's then followed as PEI-PEGn-PO $m$, with $n=1,3$ and $m=12$, 24. The different polymers synthesized and their respective $\Sigma b$ values are summarized in Table 1. All the NR measurements were made in NRW at $\mathrm{pH}$. The $\mathrm{pH}$ was adjusted by the addition of $\mathrm{NaOH}$ and $\mathrm{HCl}$. A polymer concentration of $20 \mathrm{ppm}$ was used in all the measurements. All the glassware and Teflon sample troughs were cleaned in Decon 90 solution and rinsed thoroughly in UHQ water. $\mathrm{D}_{2} \mathrm{O}$ was obtained from Sigma-Aldrich, and UHQ water was used in all the solution preparations. All the prepared solutions were clear and showed no visual indication of phase separation or precipitation.

\section{RESULTS AND DISCUSSION}

(i). Surface Tension Data. The surface tension variation with SDS concentration for SDS/PEI-PEG1-PO24 and SDS/ PEI-PEG3-PO24 at $\mathrm{pH} 7$ are shown in Figure 2. For comparison the surface tension for SDS in the absence of polymer is also shown.
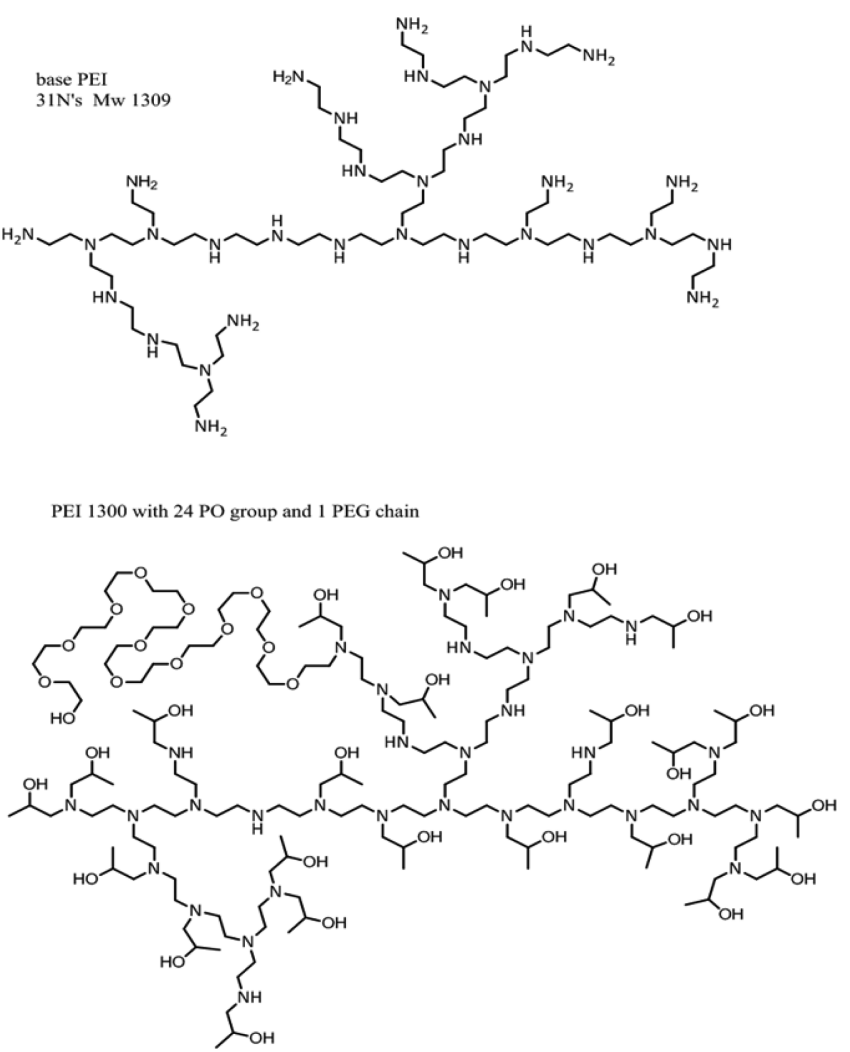

Figure 1. Structure of base PEI and PEI-PEG1-PO24.

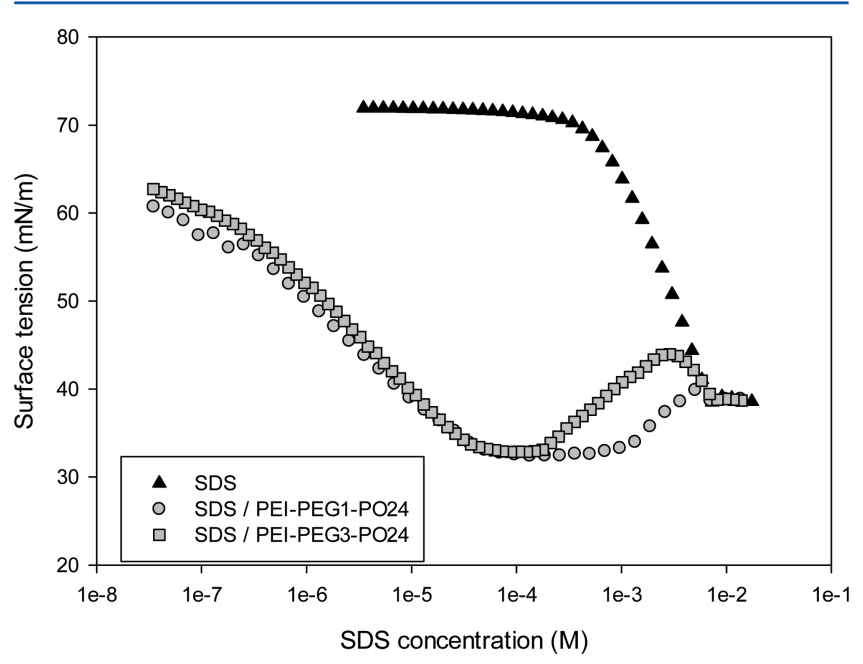

Figure 2. Surface tension as a function of SDS concentration for 20 ppm PEI-PEG1-PO24/SDS (o), PEI-PEG3-PO24/SDS ( $\square$ ), and SDS $(\Delta)$ at $\mathrm{pH} 7$.

The addition of the modified PEI to SDS results in a significant shift in the initial decrease in the surface tension to lower SDS concentrations, which is largely independent of the two polymers measured here. This is consistent with the general observation in a range of polyelectrolyte/surfactant mixtures. ${ }^{5,7}$ It arises from the strong surface interaction and surface complex formation between the surfactant and polymer, and results in a significant increase in the surface activity. With increasing surfactant concentration there is the onset of a plateau in the surface tension at a surface tension value significantly lower than the surface tension for pure SDS at the cmc; and is another indication of the enhanced surface activity. 
At higher SDS concentrations the surface tension increases toward the value for SDS at the cmc. Immediately before the $\mathrm{cmc}$ there is a peak in the surface tension which is more pronounced for PEI-PEG1-PO24 than for PEI-PEG3-PO24. At the lower SDS concentrations, $\leq 10^{-7} \mathrm{M}$, the ST is likely to be affected by depletion effects as discussed recently by $\mathrm{Li}$ et $\mathrm{al}^{42,43}$ but this does not impact upon the qualitative interpretation of that data presented here. Penfold et al. ${ }^{28}$ reported similar surface tension behavior for SDS with a $2 \mathrm{kDa}$ MW branched PEI at $\mathrm{pH} 7$ and 10, see Figure 4 in ref 28. The surface tension response reported by Zhang et al. ${ }^{33}$ in Figure 1 in that paper for SDS with PEI-EO1 or PEI-PO1 (with one EO or PO group attached to each amine group) is also broadly similar to the data in Figure 2 above.

As described in the Introduction and discussed more extensively by Bahramain et $\mathrm{al}^{7}$ the occurrence of a peak in the surface tension or an extended plateau region depends upon the competition between the surface complex formation and bulk complex formation, and the nature of the of the bulk precipitated or coacervated phase that is in equilibrium with the surface and which occurs around the region of charge neutralization. For a nondissociable precipitate the surface tension is characterized by a pronounced peak due to depletion from the surface, and is illustrated most prominently in SDS/ polydmdaac mixtures. ${ }^{10}$ For a bulk phase that is partially dissociated the surface tension behavior is characterized by a plateau region and generally an absence of a peak in the surface tension, and has been observed in a variety of different polymer/surfactant mixtures. ${ }^{5,11}$ The low surface tension plateau is a result of the surface being wetted by a more surface active concentrated phase and is often characterized by surface multilayer formation. It was shown in the $C_{n} T A B / P S S$ mixtures ${ }^{5}$ that there was a distinct transition from one characteristic surface behavior to the other as the alkyl chain length of the surfactant increases. Hence modifying the polymer-surfactant interaction by changing the surfactant structure or the structure/architecture of the polymer can tailor the surface properties. The surface tension data presented here imply that the behavior of the modified PEI/SDS mixtures is at the transition between the two extremes outlined above. Hence it might be expected that elements of both types of behavior may occur. The variation in the adsorption and the adsorbed layer structure derived from the neutron reflectivity data are hence crucial in being able to demonstrate and quantify this.

(ii). Neutron Reflectivity Data. To obtain the adsorbed amounts of SDS and polymer for all the SDS/polymer combinations measured the NR data are well described by a single monolayer with a thickness $\sim 28 \pm 2 \AA$ for SDS concentrations $<0.1 \mathrm{mM}$ and $\sim 22 \pm 2 \AA$ for SDS concentrations $>0.1 \mathrm{mM}$. Some typical NR data and associated model fits are shown in figure $S 1$ in the Supporting Information. As described in the Experimental Details, from the reflectivity data for the isotopic combinations of $\mathrm{dh}$, hd or $\mathrm{dd}$, hd the adsorbed amounts for both the SDS and polymer are obtained using eq 1. For each isotopic combination the reflectivity is modeled separately as a single layer to obtain a thickness $\mathrm{d}$ and a scattering length density $\rho$, and the product $\mathrm{d}$ $\times \rho$ is the key factor which is directly related to the adsorbed amount via eq 1 , as discussed in detail elsewhere. ${ }^{37}$ The variation in the SDS and polymer adsorption with SDS concentration are shown in Figure 3 for SDS/PEI-PEG1-PO12, SDS/PEI-PEG1-PO24, and for SDS in the absence of polymer, and the key parameters are summarized in Table 2.

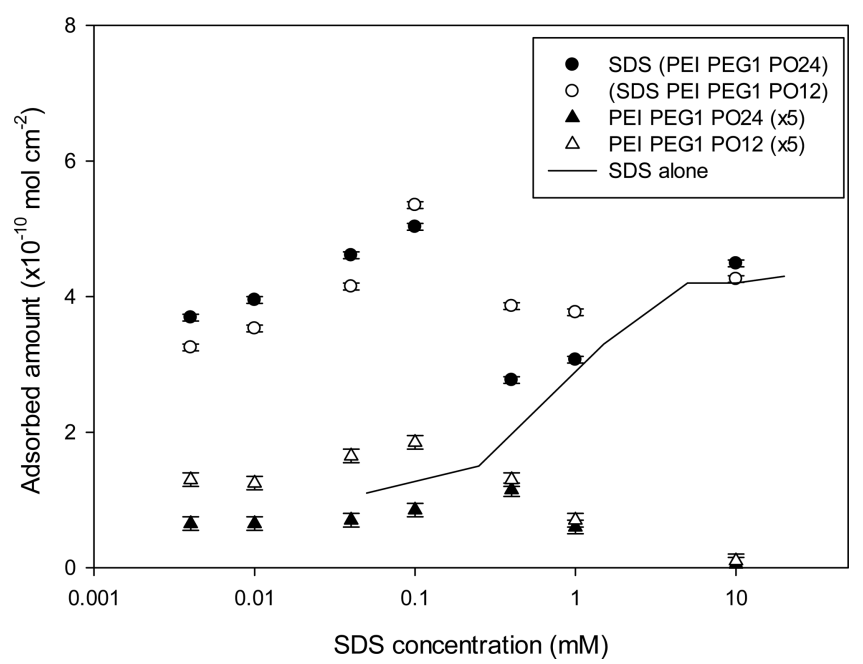

Figure 3. Adsorbed amount versus SDS concentration for SDS/PEIPEG1-PO12, SDS/PEI-PEG1-PO24, and SDS; see legend for details. The adsorbed amount of polymer is display $\times 5$ for clarity.

Table 2. Adsorption Data for SDS/PEI-PEG1-PO12 and SDS/PEI-PEG1-PO24

\begin{tabular}{|c|c|c|c|c|}
\hline \multicolumn{5}{|c|}{ (a) SDS/PEI-PEG1-PO12 } \\
\hline \multirow[b]{2}{*}{$\begin{array}{l}\text { SDS conc } \\
(\mathrm{mM})\end{array}$} & \multicolumn{2}{|r|}{ SDS } & \multicolumn{2}{|r|}{ polymer } \\
\hline & $\begin{array}{c}A \\
\left( \pm 2 \AA^{2}\right)\end{array}$ & $\begin{array}{c}\Gamma\left( \pm 0.05 \times 10^{-10}\right. \\
\mathrm{mol} \mathrm{cm}\end{array}$ & $\begin{array}{c}A \\
\left( \pm 2 \AA^{2}\right)\end{array}$ & $\begin{array}{c}\Gamma\left( \pm 0.02 \times 10^{-10}\right. \\
\left.\mathrm{mol} \mathrm{cm} \mathrm{cm}^{-2}\right)\end{array}$ \\
\hline 10 & 39 & 4.26 & $>10000$ & $<0.01$ \\
\hline 1 & 44 & 3.77 & 1297 & 0.13 \\
\hline 0.4 & 43 & 3.86 & 643 & 0.26 \\
\hline 0.1 & 31 & 5.35 & 451 & 0.37 \\
\hline 0.04 & 40 & 4.15 & 501 & 0.33 \\
\hline 0.01 & 42 & 3.95 & 653 & 0.25 \\
\hline 0.004 & 51 & 3.25 & 631 & 0.26 \\
\hline \multicolumn{5}{|c|}{ (b) SDS/PEI-PEG1-PO24 } \\
\hline & \multicolumn{2}{|r|}{ SDS } & \multicolumn{2}{|r|}{ polymer } \\
\hline $\begin{array}{l}\text { SDS conc } \\
(\mathrm{mM})\end{array}$ & $\begin{array}{c}A \\
\left( \pm 2 \AA^{2}\right)\end{array}$ & $\begin{array}{c}\Gamma\left( \pm 0.05 \times 10^{-10}\right. \\
\mathrm{mol} \mathrm{cm}\end{array}$ & $\begin{array}{c}A \\
\left( \pm 2 \AA^{2}\right)\end{array}$ & $\begin{array}{c}\Gamma\left( \pm 0.02 \times 10^{-10}\right. \\
\mathrm{mol} \mathrm{cm} \\
-2)\end{array}$ \\
\hline 10 & 37 & 4.44 & $>10000$ & $<0.01$ \\
\hline 1 & 54 & 3.07 & 1390 & 0.12 \\
\hline 0.4 & 60 & 2.77 & 724 & 0.23 \\
\hline 0.1 & 33 & 5.03 & 987 & 0.17 \\
\hline 0.04 & 36 & 4.61 & 1179 & 0.14 \\
\hline 0.01 & 47 & 3.53 & 1307 & 0.13 \\
\hline 0.004 & 45 & 3.69 & 1311 & 0.13 \\
\hline
\end{tabular}

At low surfactant concentrations the SDS adsorption is enhanced significantly relative to the SDS adsorption in the absence of polymer. The adsorption increases up to a maximum at an SDS concentration $\sim 0.1 \mathrm{mM}$. Between SDS concentrations of 0.1 and $1 \mathrm{mM}$ the amount of SDS at the interface decreases markedly until at SDS concentrations $>1 \mathrm{mM}$ it starts to increase again toward the value for SDS in the absence of polymer and above the SDS cmc. Between the SDS concentrations of 1 and $10 \mathrm{mM}$ the amount of SDS at the interface is comparable to that in the absence of polymer. The amount of polymer at the interface also increases as the SDS concentration increases up to a maximum which coincides with the maximum in the SDS adsorption. Over that range of SDS concentrations the mole ratio of surfactant/polymer is approximately constant, and we will return to this point later 
in the discussion. At higher SDS concentrations $(>0.1 \mathrm{mM})$ the amount of polymer at the interface decreases until at an SDS concentration of $10 \mathrm{mM}$ it is immeasurably small. The SDS concentration region where the SDS is partially depleted from the surface and the polymer adsorption decreases toward zero coincides with the surface tension region where the broad peak in the surface tension occurs. Both observations are consistent with the depletion from the surface in favor of bulk aggregate formation. The subsequent increase in the SDS adsorption is as a result of the free SDS monomer concentration increasing as the bulk complexes become saturated in surfactant. These trends are broadly consistent with the observations previously reported for SDS/polydmdaac mixtures. ${ }^{10}$ In Figure 3 the adsorption data for two different SDS/polymer mixtures are compared. The difference in the two modified PEI's compared is the degree of propoxylation. The amount of polymer adsorption is greater for PEI-PEG1-PO12 compared to PEIPEG1-PO24 at SDS concentrations $<0.1 \mathrm{mM}$. However, the surface depletion of the SDS is larger in the presence of PEIPEG1-PO24 than for PEI-PEG1-PO12. This indicates that the bulk complex formation is more favored with PEI-PEG-PO24 than with PEI-PEG1-PO12. At the lower SDS concentrations the amount of SDS adsorption is slightly higher for PEI-PEG1PO24 than for PEI-PEG1-PO12. Measurements of the surface adsorption of the polymers in the absence of surfactant indicate that PEI-PEG1-PO12 is more surface active than PEI-PEG1$\mathrm{PO} 24$, with an adsorbed amount of $0.24 \times 10^{-10} \mathrm{~mol} \mathrm{~cm}^{-2}$ compared to $0.12 \times 10^{-10} \mathrm{~mol} \mathrm{~cm}^{-2}$ for PEI-PEG1-PO24.

Figure 4 shows the variation in SDS and polymer adsorption with SDS concentration for the combinations SDS/PEI-PEG3-

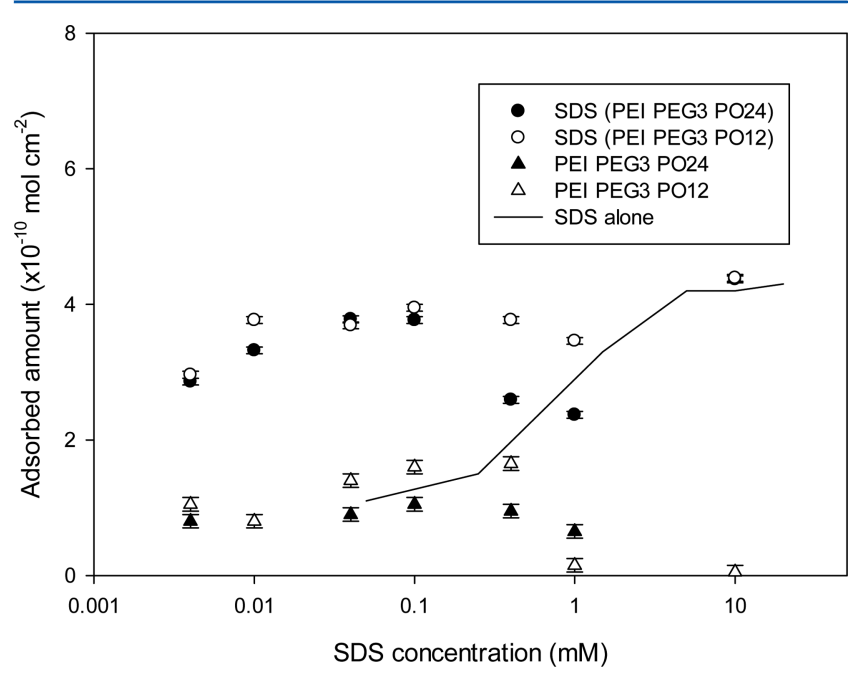

Figure 4. Adsorbed amount versus SDS concentration for SDS/PEIPEG3-PO12, SDS/PEI-PEG3-PO24, and SDS; see legend for details. The adsorbed amount of the polymer is displayed as $\times 5$ for clarity.

PO12, and SDS/PEI-PEG3-PO24, and SDS in the absence of polymer. The patterns of adsorption follow the same broad trends as illustrated in Figure 3 for the modified PEI with only one PEG chain attached. The associated parameters are summarized in table S1 in the Supporting Information. For the adsorption data presented in Figure 4 increasing the degree of propoxylation has relatively little impact upon the SDS or polymer adsorption. The only significant difference is, as was observed in the data in Figure 3, the partial depletion from the surface at SDS concentrations $\sim 1 \mathrm{mM}$ is greater for the polymer with the higher degree of propoxylation. The differences in the polymer adsorption with the degree of propoxylation are also less pronounced compared to the data in Figure 3. That is, changing the degree of propoxylation has less impact on the pattern of adsorption, and this is in part due to the greater influence of the larger number of PEG chains attached. This is also consistent with the intrinsic surface activity of the polymers in the absence of surfactant, where the adsorbed amount for PEI-PEG3-PO12 and PEI-PEG3-PO24 in the absence of surfactant is $\sim 0.14 \times 10^{-10} \mathrm{~mol} \mathrm{~cm}{ }^{-2}$ and is independent of the degree of propoxylation. Compared particularly to the adsorption for PEI-PEG1-PO12 the larger number of attached PEG chains makes the polymer more soluble and less surface active. The adsorbed amount of SDS at the interface for SDS concentrations $<0.1 \mathrm{mM}$ in the presence of PEI-PEG3-PO12(PO24) is also reduced compared to the PEI-PEG1-PO12(PO24) polymers, and is indicative of a reduced surface interaction between the SDS and polymer. Figure S2, and Table S3 in the Supporting Information summarize the adsorption data for SDS/PEI-PO12. In the absence of any attached PEG chains the broad trends in the adsorption are similar to those presented in Figures 3 and 4 for the polymer with one or three PEG chains attached. Notably, however, the intrinsic surface activity of this modified PEI is much less than the other modified PEI's studied here, with an adsorbed amount $\sim 0.05 \times 10^{-10} \mathrm{~mol} \mathrm{~cm} \mathrm{~cm}^{-2}$.

(iii). Discussion. The enhanced SDS adsorption down to relatively low SDS concentrations, shown in Figures 3 and 4, is broadly similar to that reported in other modified PEI/SDS ${ }^{33,34}$ mixtures, but not as pronounced as that reported in PEI/SDS mixtures. ${ }^{28}$ The enhancement in the SDS adsorption is broadly independent of the different polymer architectures explored in this study, and arises from the strong surface interaction or complexation between the SDS and polymer. As was observed by Zhang et al. $^{33}$ the addition of a single ethylene oxide or propylene group did not substantially weaken the SDS polymer interaction and the enhanced surface adsorption, and this is also observed for the PEI-PEG $-\mathrm{PO}_{\mathrm{m}}$ polymers studied here. However, Zhang et al. ${ }^{33}$ did report that the adsorption was slightly reduced for $\mathrm{PEI}_{-\mathrm{EO}_{1}}$ than for $\mathrm{PEI}-\mathrm{PO}_{1}$, and attributed this to a balance between the steric hindrance associated with attached ethylene oxide or propylene oxide group and their relative hydrophobicities. As previously reported $^{34}$ the presence of a single or three ethylene oxide chains has little impact upon the adsorption properties, and the results are hence entirely consistent with those previous observations. $^{33,34}$

Apart from the important aspect of exploring the different polymer architectures, and especially the effect of attachment of the propylene oxide groups, an important contribution of this study is the ability to determine the amount of SDS and polymer at the interface, arising from the deuterium labeling of both the surfactant and polymer. Some selected adsorbed amounts of polymer and SDS and their ratios are summarized in Table 3.

As previously discussed the surface composition, ratio of SDS/polymer, is approximately constant in the SDS concentration range 0.004 to $0.1 \mathrm{mM}$, and the data for SDS concentrations of 0.004 and $0.1 \mathrm{mM}$ are shown in Table 3 . For the PEI-PEG1-POn/SDS mixtures the mole ratio of SDS/ polymer varies with the degree of propoxylation of the polymer.

Indeed the mole ratio is approximately equal to the degree of propoxylation. For the PEI-PEG3-POn polymer the mole ratio 
Table 3. Adsorbed Amounts of SDS and Polymer

\begin{tabular}{|c|c|c|c|c|}
\hline surfactant concentration $(\mathrm{mM})$ & polymer & $\Gamma_{\mathrm{SDS}}\left( \pm 0.05 \times 10^{-10} \mathrm{~mol} \mathrm{~cm}^{-2}\right)$ & $\Gamma_{\text {polymer }}\left( \pm 0.05 \times 10^{-10} \mathrm{~mol} \mathrm{~cm}^{-2}\right)$ & $\Gamma_{\text {sds }} / \Gamma_{\text {polymer }}$ \\
\hline 0.1 & PEI-PEG1-PO12 & 5.35 & 0.37 & 15 \\
\hline 0.1 & PEI-PEG1-PO24 & 5.03 & 0.17 & 30 \\
\hline 0.1 & PEI-PEG3-PO12 & 3.95 & 0.32 & 12 \\
\hline 0.1 & PEI-PEG3-PO24 & 3.72 & 0.21 & 18 \\
\hline 0.004 & PEI-PEG1-PO12 & 3.25 & 0.26 & 13 \\
\hline 0.004 & PEI-PEG1-PO24 & 3.69 & 0.13 & 30 \\
\hline 0.004 & PEI-PEG3-PO12 & 2.96 & 0.21 & 14 \\
\hline 0.004 & PEI-PEG3-PO24 & 2.86 & 0.16 & 18 \\
\hline
\end{tabular}

Table 4. Variation in Layer Thickness with SDS Concentration and "Contrast" for SDS/PEI-PEG1-PO12 (PO24) Mixtures

\begin{tabular}{|c|c|c|c|c|c|c|}
\hline \multirow[b]{2}{*}{ polymer } & \multirow[b]{2}{*}{ SDS concentration $(\mathrm{mM})$} & \multirow[b]{2}{*}{ SDS area/molecule $\left( \pm 2 \AA^{2}\right)$} & \multicolumn{3}{|c|}{ layer thickness } & \multirow[b]{2}{*}{ mean layer thickness (from $\mathrm{dh}, \mathrm{dd})( \pm 2 \AA)$} \\
\hline & & & $\mathrm{dh}( \pm 2 \AA)$ & hd $( \pm 5 \AA)$ & $\mathrm{dd}( \pm 2 \AA)$ & \\
\hline $\mathrm{PO} 24$ & 10.0 & 37 & 24 & $a$ & 24 & 24 \\
\hline $\mathrm{PO} 24$ & 0.1 & 33 & $36^{b}$ & 36 & $28^{b}$ & 32 \\
\hline $\mathrm{PO} 24$ & 0.01 & 47 & 30 & 32 & 26 & 28 \\
\hline $\mathrm{PO} 24$ & 0.004 & 45 & 32 & 30 & 26 & 29 \\
\hline PO12 & 10.0 & 39 & 22 & $a$ & 19 & 21 \\
\hline $\mathrm{PO} 12$ & 0.1 & 31 & $36^{b}$ & 35 & $24^{b}$ & 30 \\
\hline PO12 & 0.01 & 42 & 26 & 32 & 20 & 23 \\
\hline
\end{tabular}

${ }^{a}$ No measurable polymer adsorption. ${ }^{b}$ Modeled in detail as two layers (see Table 5).

of surfactant/polymer also varies with the degree of propoxylation. For PEI-PEG3-PO12 the ratio is similar to that for PEI-PEG1-PO12. For PEI-PEG3-PO24 the ratio is larger than for PEI-PEG3-PO12, and no longer reflects the degree of propoxylation. From previous studies by Penfold et al. $^{28}$ and Zhang et al. ${ }^{33}$ on the surface complex formation, from the patterns of adsorption SDS molecules bind to available amine groups and to amine groups with a single PO group attached with a broadly similar affinity. Specifically for the SDS/ PEI-PO1 mixtures, ${ }^{33}$ where SDS/polymer ratios were determined, at similar conditions to those reported here the mole ratio of surfactant to degree of propoxylation varies from a ratio that is half the degree of propoxylation at low SDS concentrations to a value similar to the degree of propoxylation at higher surfactant concentrations. Unlike the unmodified PEI the attachment of the EO chain and the propoxylation makes the modified PEI surface active in the absence of SDS. As discussed in the following paragraphs the surface activity of the modified PEI in the absence of surfactant varies with the number of EO chains attached and with the degree of propoxylation. The amount of polymer at the interface is now associated with the intrinsic surface activity of the polymer, how that is affected by the binding of the SDS and the competition between the now three surface active species, in a complex way. Hence the variations in SDS/polymer ratio reported here reflect that complex competition, and without a specific model it is difficult to predict.

Zhang et al. ${ }^{33}$ reported the adsorption of the modified PEI's, PEI-EO3, PEI-EO1, and PEI-PO1 at the air-water interface, with adsorbed amounts of $0.12,0.14$, and $0.11 \times 10^{-10} \mathrm{~mol}$ $\mathrm{cm}^{-2}$, respectively, at $\mathrm{pH} 7$. An increase in the surface activity of the modified PEI's with increasing $\mathrm{pH}$ as the charge on the PEI decreased was reported; although the difference between the values at $\mathrm{pH} 7$ and 10 was negligible. The modified PEI's studied here have similar intrinsic surface activities (see Table $\mathrm{S} 3$ in the Supporting Information), at $\mathrm{pH} 7$, in that PEI-PEG1PO24, PEI-PEG3-PO24, and PEI-PEG3-PO12 have adsorbed amounts of $0.12,0.14$, and $0.15 \times 10^{-10} \mathrm{~mol} \mathrm{~cm}{ }^{-2}$, respectively.
In contrast the surface activity of PEI-PEG1-PO12 is larger, $0.25 \times 10^{-10} \mathrm{~mol} \mathrm{~cm}^{-2}$.

When three ethylene oxide chains are attached changing the degree of propoxylation has little impact upon the surface activity of the polymer. With one ethylene oxide chain attached the degree of propoxylation has a significant impact upon the surface activity of the polymer, and for the lower amount of propylene oxide it is much larger. Notably, however, the modified polymer, PEI-PO12, with no ethylene oxide chain attached has the lowest surface activity, $0.05 \times 10^{-10} \mathrm{~mol} \mathrm{~cm}{ }^{-2}$; and it is noted that PEI alone is not surface active. ${ }^{28}$ Although ethoxylation or propoxylation confers a degree of surface activity on PEI, the relative surface activity of the differently modified polymers is a balance between the changes in solubility due to ethoxylation/propoxylation, the greater hydrophobicity of the PO groups relative to EO groups and the steric constraints due to the larger attached PO groups. These trends correlate directly with the relative amounts of polymer adsorbed in the polymer/surfactant mixtures explored here. The trends also correlate with the extent of the surface depletion, in that the surface depletion is greater with the less surface active polymers.

Over most of the SDS concentration range explored the NR data are adequately modeled as a single layer of adsorbed surfactant-polymer complex at the interface, and the focus was on obtaining the product $\mathrm{d} \times \rho$ for the determination of the adsorbed amounts, as described earlier. However, for the NR data for SDS/PEI-PEG1-PO24 and SDS/PEI-PEG1-PO12 a more detailed evaluation of the surface structure has been obtained. From the systematic variation in the statistical chisquared parameter with layer thickness using the conventional weighted least-squares minimization for a single layer model in $\rho$ is the refined parameter, a more accurate estimate of the layer thickness is obtained. The mean thicknesses obtained are summarized in Table 4. A series of typical chi-squared variations with layer thickness are shown in Figure S3 in the Supporting Information for $0.01 \mathrm{mM}$ SDS/PEI-PEG1-PO12. 
For both the PEI-PEG1-PO24 and PEI-PEG1-PO12 polymers there are some notable trends in the variation in the surface layer thickness in Table 4. At $10 \mathrm{mM}$ SDS, where there is only SDS at the surface, the layer is consistently thinner, in the range 21 to $24 \AA$. At lower SDS concentrations, 0.01 and $0.004 \mathrm{mM}$, the surface layer is substantially thicker, in the range 23 to $28 \AA$. This reflects the contribution of the polymer and its organization with respect to the SDS at the interface. When the modified PEI is present at the interface with the SDS the adsorbed layer is systematically thicker. This was not previously observed for the SDS/PEI mixtures, ${ }^{28}$ for 25 $\mathrm{kDa}$ linear and branched PEI's and for $2 \mathrm{kDa}$ branched PEI. In that case, a mean adsorbed layer thickness $\sim 21 \AA$ was reported for SDS and SDS/PEI adsorption at the interface. Similar observations were made for SDS/PEI-PO ${ }_{1}$ (for a $2 \mathrm{k} \mathrm{Da}$ branched PEI) where a mean adsorbed layer thickness $\sim 20 \AA$ was reported. ${ }^{33}$ In that study, Zhang et al. ${ }^{33}$ reported an increase in the adsorbed layer thickness from $\sim 20 \AA$ for SDS to $\sim 27 \AA$ for SDS/PEI-EO 3 mixtures. In the results summarized here in Table 5 the mean thickness increases from $\sim 21 \AA$ for

Table 5. Two-Layer Model Fit Parameters for SDS/PEIPEG1-PO12 (PO24) at an SDS Concentration of $0.1 \mathrm{mM}$

\begin{tabular}{cccccc} 
polymer & “contrast” & $\begin{array}{c}d_{1} \\
( \pm 2 \AA)\end{array}$ & $\begin{array}{c}\rho_{1} \\
\left( \pm 0.05 \times 10^{-6}\right.\end{array}$ & $\begin{array}{c}d_{2} \\
\AA^{-2}\end{array}$ & $\begin{array}{c}\rho_{2} \\
( \pm 2 \AA)\end{array}$ \\
PO24 & $\mathrm{dh}$ & 26 & 3.2 & 32 & 0.8 \\
PO24 & $\mathrm{dd}$ & 24 & 3.8 & 34 & 0.4 \\
PO12 & $\mathrm{dh}$ & 26 & 3.0 & 21 & 1.0 \\
PO12 & $\mathrm{dd}$ & 18 & 4.5 & 18 & 0.75 \\
\hline
\end{tabular}

SDS alone at the interface to $\sim 23 \AA$ for SDS/PEI-PEG1-PO12. Although within error there is a systematic increase $\sim 2 \AA$ due to the presence of the polyelectrolyte at the interface. Allowing a dimension $\sim 3 \AA$ for the extent of the propylene oxide group this implies that the extra thickness is accounted for by the presence of the propylene oxide group. This implies that the polyelectrolyte adopts a relatively flat conformation against the surface as defined by the surfactant layer, similar to the conformation against a charged solid surface. ${ }^{42}$ In contrast, for the SDS/PEI-PEG1-PO24 mixture the adsorbed layer thickness increases to mean thickness $\sim 28$ to $29 \AA$ due to the presence of the polyelectrolyte at the interface. This is now systematically larger than the size of the propylene oxide group. For the lower degree of propoxylation there are sufficient free amine groups on the polyelectrolyte chain for the polyelectrolyte - SDS interaction to be directly between the sulfate-amine nitrogen interaction. Hence the conformation of the polyelectrolyte at the interface for SDS/PEI-PEG1-PO12 is similar to that observed for PEI/SDS. ${ }^{28}$ For the SDS/PEI-PEG1-PO24 mixture most of the amine nitrogen's are now associated with the attached propylene oxide groups or the poly(ethylene oxide) chain. The increased thickness, over and above that accounted for by the presence of a single propylene oxide group, observed in that case implies that the conformation of the polyelectrolyte at the interface has altered to accommodate the SDS interaction with the bulkier propylene oxide groups.

For both polymers at an SDS concentration of $0.1 \mathrm{mM}$ the data for the "contrasts" $\mathrm{dh}$ and dd are best modeled by two layers; whereas the "contrast" hd is still relatively well described by a single thicker layer. For the "contrasts" dh and dd the two layer model parameters are summarized in Table 5. Each layer is characterized by a thickness $\mathrm{d}$ and a scattering length density $\rho$, and where layer 1 is the layer adjacent to the air phase.

Similar observations were made by Batchelor et al., ${ }^{34}$ where some modest layering was observed in a limited range of SDS concentrations and solution $\mathrm{pH}$. In that case at $\mathrm{pH} 10$ it was more pronounced, but at $\mathrm{pH} 7$ structures similar to those reported here were observed. Similar two-layer structures were also reported in other polyelectrolyte/surfactant mixtures; and notably for PEI/SDS ${ }^{28}$ and modified PEI/SDS mixtures. ${ }^{33}$ The work of Penfold et al. ${ }^{28}$ and Zhang et al. ${ }^{33}$ showed that the two layer structures were often a precursor to the formation of more well-defined extended surface layered structures such as a welldefined surface bilayer or multilayer structures.

The region of partial surface depletion, between SDS concentrations of 0.1 to $10 \mathrm{mM}$, was also reported by Batchelor et al., ${ }^{34}$ but the effect is more pronounced here. Similar patterns of adsorption were reported in other polymer/surfactant mixtures, and notably in mixtures such as SDS/polydmdaac. ${ }^{10}$ The notable difference between those systems and the results presented here for the SDS/PEI-PEG-PO mixtures is that there is no accompanying evidence for precipitation/coacervation in the depletion region for SDS/PEI-PEG-PO, whereas the mixtures such as SDS/polydmdaac exhibit precipitation phenomena around the region of charge neutralization. The form of the partial surface depletion in favor of bulk complex formation does show some distinct trends with the changing polymer architecture. Increasing the amount of propylene oxide attached (from 12 to $24 \mathrm{~mol}$ ) increases the amount of surface depletion. Increasing the number of ethylene oxide chains attached from 1 to 3 chains has a similar impact on the surface depletion.

In the thermodynamically consistent model of the surface adsorption in polyelectrolyte/ionic surfactant mixtures at the air-water interface Bahramian et al. ${ }^{7}$ describe two different characteristic behaviors which are intrinsically linked to the nature of bulk polymer/surfactant complex formation and the competition with surface complexation. For nondissociated bulk complexation surface depletion occurs, and can result in a peak on the surface tension. For dissociable or partially dissociable bulk complex formation a broad plateau in the surface tension with a relatively low interfacial tension occurs, and surface multilayer formation often occurs. The results reported by Zhang et al. ${ }^{33}$ for SDS/PEI-PO 1 (and PEI-EO ${ }_{1}$ ) show a transition from a surface tension behavior characterized by a plateau of low surface tension to a peak in the surface tension as the $\mathrm{pH}$ decreases from 10 to 7 to 3 . The peak in the surface tension at low $\mathrm{pH}$ is more pronounced for PEI-EO . The surface tension reported for SDS/PEI-PO ${ }_{1}$ at $\mathrm{pH} 7$ and 10 is similar to that reported here for PEI-PEG1-PO12 (PO24) in Figure 2. The corresponding surface adsorption from the work of Zhang et al. ${ }^{33}$ shows that although significant surface

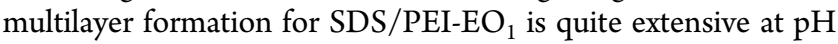
7 and 10, it is only weakly present in a narrow regime at $\mathrm{pH} 10$

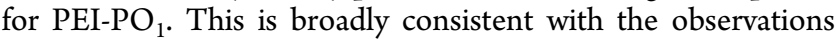
for the PEI-PEGn-POm polymers studied here, in that a weak surface ordering is observed at $\mathrm{pH} 7$ in a narrow range of SDS concentrations. Hence the NR and ST results presented here indicate that the PEI (modified by the addition of a limited number of ethylene oxide chains and propylene oxide groups)/ SDS mixtures are at the transition between those two extremes of behavior. That is, surface depletion effects and the onset of surface ordering both occur. Furthermore, this occurs in circumstances where due to the nature of the ethoxylation of 
the PEI there are no effects due to precipitation, and the solutions remain clear throughout the vicinity of charge neutralization.

\section{CONCLUSIONS}

The ethoxylation of PEI by the addition of ethylene oxide chains in discrete blocks and the addition of propylene groups to the available amine groups modifies the polyelectrolyte interaction with the anionic surfactant SDS such that the adsorption properties are at the transition between the extremes of behavior attributed to polyelectrolyte/ionic surfactant mixtures. ${ }^{7}$ As such enhanced adsorption at low SDS concentrations, nascent surface layering, and partial surface depletion close to charge neutralization are all observed. The nature of the modification to the PEI, as previously demonstrated, ${ }^{34}$ sufficiently increases the solubility such that precipitation effects are not observed around charge neutralization. The number of propylene oxide groups added affects the enhancement in the adsorption, whereas the number of ethylene oxide chains added has less of an effect on the adsorption. Increasing the degree of propoxylation and ethoxylation both affect the depletion region. The results demonstrate how modifying the polymer structure can be used to fine-tune both the solution and surface properties and optimize the favorable features of both.

\section{ASSOCIATED CONTENT}

\section{S Supporting Information}

The Supporting Information is available free of charge on the ACS Publications website at DOI: 10.1021/acs.langmuir.5b04419.

Tables and figures of some of the adsorption data (PDF)

\section{AUTHOR INFORMATION}

\section{Corresponding Author}

*E-mail: jeff.penfold@stfc.ac.uk.

\section{Author Contributions}

All the authors have given their approval for the submitted manuscript.

\section{Notes}

The authors declare no competing financial interest.

\section{ACKNOWLEDGMENTS}

We acknowledge the provision of neutron beam time on the INTER (ISIS) reflectometer, and the scientific and technical support of the Instrument Scientists and technical staff.

\section{REFERENCES}

(1) Jones, N. M. Interaction of sodium dodecyl sulfate with polyethylene oxide. J. Colloid Interface Sci. 1967, 23, 36-42.

(2) Goddard, E. D.; Ananthapadamanbhan, K. P. Interaction of surfactants with polymers and proteins; CRC Press: Boca Raton, FL, 1993.

(3) Polymer-surfactant systems; Kwak, J. C. T., Ed.; Surfactant Science Series; Marcel Dekker: New York, 1998; Vol 77.

(4) Langevin, D. Polyelectrolyte and surfactant mixed solutions. Behaviour at surfaces and in thin films. Adv. Colloid Interface Sci. 2001, 89, 467-484.

(5) Taylor, D. J. F.; Thomas, R. K.; Penfold, J. Polymer-surfactant interactions at the air-water interface. Adv. Colloid Interface Sci. 2007, 132, 69-110.

(6) Cabane, B.; Duplessix, R. Neutron scattering of water soluble polymers and charged surfactants. Colloids Surf. 1985, 13, 19-33.
(7) Bahramian, A.; Thomas, R. K.; Penfold, J. The adsorption behaviour of ionic surfactants and their mixtures with nonionic polymers and polyelectrolytes of opposite charge at the air-water interface. J. Phys. Chem. B 2014, 118, 2769-2783.

(8) Goddard, E. D. Polymer-surfactant interactions: Interfacial aspects. J. Colloid Interface Sci. 2002, 256, 228-235.

(9) Goddard, E. D. Polymer-surfactant interactions, Part II, Polymers and surfactants of opposite charge. Colloids Surf. 1986, 19, 301-329.

(10) Staples, E.; Tucker, I.; Penfold, J.; Warren, N.; Thomas, R. K.; Taylor, D. J. F. Organisation of polymer-surfactant mixtures at the airwater interface: sodium dodecyl sulfate and poly(dimethyldiallylammonium chloride). Langmuir 2002, 18, 5147-5153.

(11) Taylor, D. J. F.; Thomas, R. K.; Penfold, J. The adsorption of oppositely charged polyelectrolyte-surfactant mixtures: neutron reflection from dodecyltrimethyl ammonium bromide and sodium poly(styrene sulfonate) at the air-water interface. Langmuir 2002, 18, $4748-4757$.

(12) Chou, S. I.; Bae, J. H. Surfactant precipitation and redissolution in brine. J. Colloid Interface Sci. 1983, 96, 192-203.

(13) Steller, K. L.; Scamehorn, J. F. Surfactant precipitation in aqueous solutions containing mixtures of anionic and nonionic surfactants. J. Am. Oil Chem. Soc. 1986, 63, 566-574.

(14) Scamehorn, J. F.; Christian, S. D.; Ellington, R. T. In Surfactant based separation processes; Scamehorn, J. F., Harwell, J. H., Eds.; Surfactant Science Series; Marcel Dekker: New York, 1989; Vol 33.

(15) Somersundaran, P.; Ananthapadmanbhan, K. P.; Celik, M. S. Precipitation and redissolution phenomena in sulfonate $-\mathrm{ACl}_{3}$ solutions. Langmuir 1988, 4, 1061-1063.

(16) Paton Morales, P.; Talens-Alesson, F. I. Effect of ionic strength and competitive adsorption of $\mathrm{Na}^{+}$on flocculation of Lauryl sulfate micelles with $\mathrm{Al}^{3+}$. Langmuir 2001, 17, 6059-6064.

(17) Scheilbel, J. J. The evolution of anionic surfactant technology to meet the requirements of the laundry detergent industry. J. Surfactants Deterg. 2004, 7, 319-328.

(18) Yangxin, Y. U.; Zhao, J.; Bayley, A. E. Development of surfactants and builders in detergent formulations. Chin. J. Chem. Eng. 2008, 16, 517-527.

(19) Winnik, M. A.; Bystryak, S. M.; Chassenieux, C.; Strashko, V.; MacDonald, P. M.Study of interaction of poly(ethyleneimine) with sodium dodecyl sulfate in aqueous solution by light scattering, conductometry, NMR, and microcalorimetry. Langmuir 2000, 16, 4495-4510.

(20) Li, Y.; Ghoreishi, S. M.; Warr, J.; Bloor, D. M.; Holzwarth, J. F.; Wyn-Jones, E. Binding of sodium dodecyl sulfate to some polyethyleneimines and their ethoxylated derivatives at different $\mathrm{pH}$ values. Electromotive force and microcalorimetry studies. Langmuir 2000, 16, 3093-3100.

(21) Zintchenko, A.; Phillip, A.; Dehsharhri, A.; Wagner, E. Simple modifications of branched PEI lead to highly efficient siRNA carriers with low toxicity. Bioconjugate Chem. 2008, 19, 1448-1455.

(22) Karaman, D. S.; Gukin-Sarfraz, T.; Hedstrom, G.; Duchanyo, A.; Eklund, P.; Rosenholm, J. M. Rational evaluation of the utilization of PEI-PEG copolymers for the facilitation of silicon nanoparticulate systems in biomedical applications. J. Colloid Interface Sci. 2014, 418, 300-310.

(23) Perevyazko, I. Y.; Bauer, M.; Pavlov, G. M.; Hoeppener, S.; Schubert, S.; Fischer, D.; Schubert, U. S. Polylectrolyte complexes of DNA and linear PEI: formation, composition and properties. Langmuir 2012, 28, 16167-16176.

(24) Kim, S.; Choi, J. S.; Jang, H. S.; Juh, H.; Park, J. Hydrophobic modification of polyethyleneimine for Gene transfectant. Bull. Korean Chem. Soc. 2001, 22, 1068-1075.

(25) Liu, C.; Liu, F.; Feng, L.; Li, M.; Zhang, J.; Zhang, N. The targeted co-delivery of DNA and doxorubicin to tumor cells via multifunctional PEI-PEG based nano-particles. Biomaterials 2013, 34, 2547-2564.

(26) Godbey, W. T.; Wu, K.; Mikos, A. G. Poly(ethyleneimine) and its role in gene delivery. J. Controlled Release 1999, 60, 149-160. 
(27) Godbey, W. T.; Wu, K. K.; Mikos, A. G. Size matters, molecular weight affects the efficiency of poly(ethyleneimine) as a gene delivery vehicle. J. Biomed. Mater. Res. 1999, 45, 268-275.

(28) Penfold, J.; Tucker, I.; Thomas, R. K.; Zhang, J. Adsorption of polyelectrolyte/surfactant mixtures at the air-solution interface: polyethyleneimine/sodium dodecyl sulfate. Langmuir 2005, 21, 10061-10072

(29) Meszaros, R.; Thompson, L.; Bos, M.; Varga, I.; Gilanyi, T. Interaction of sodium dodecyl sulfate with polyethyleneimine: surfactant induced polymer solution colloidal dispersion transition. Langmuir 2003, 19, 609-615.

(30) Angelescu, D. G.; Nylander, T.; Piculell, L.; Linse, P.; Lindman, B. Adsorption of branched and linear PEI-EO conjugates on hydrophilic silica investigated by ellipsometry and Monte-Carlo simulations. Langmuir 2011, 27, 9961-9971.

(31) Angus-Smythe, A.; Bain, C. D.; Varga, I.; Campbell, R. A. Effects of bulk aggregation on PEI-SDS monolayers at the dynamic airsolution interface: depletion due to precipitation versus enrichment by a convection/spreading mechanism. Soft Matter 2013, 9, 6103-6117.

(32) Penfold, J.; Taylor, D. J. F.; Thomas, R. K.; Tucker, I.; Thompson, L. J. The adsorption of polymer/surfactant mixtures at the air-water interface: ethoxylated PEI and SDS. Langmuir 2003, 19, $7740-7745$.

(33) Zhang, X. L.; Taylor, D. J. F.; Thomas, R. K.; Penfold, J. Adsorption of polyelectrolyte/surfactant mixtures at the air-water interface: modified polyethyleneimine and sodium dodecyl sulfate. Langmuir 2011, 27, 2601-2612.

(34) Batchelor, S. N.; Tucker, I. M.; Petkov, J. T.; Penfold, J.; Thomas, R. K. Sodium dodecyl sulfate-ethoxylated polyethyleneimine adsorption at the air-water interface: how the nature of ethoxylation affects the pattern of adsorption. Langmuir 2014, 30, 9761-9769.

(35) SURF reflectometer at the ISIS facility, http://www.isis.stfc.ac. uk/instruments/SURF.

(36) INTER reflectometer at the ISIS facility, http://www.isis.stfc.ac. uk/instruments/INTER.

(37) Lu, J. R.; Thomas, R. K.; Penfold, J. Surfactant layers at the airwater interface: structure and composition. Adv. Colloid Interface Sci. 2000, 84, 143-304.

(38) Heavens, O. S. Optical properties of thin solid films; Dover Publications: New York, 1991.

(39) Lekner, J. Theory of Reflection; Martinus Nijhoff, Publishers: The Netherlands, 1987.

(40) ISIS Deuteration facility, http://www.isis.stfc.ac.uk/supportlaboratories/ISIS-isotope-facility/ISIS-deuteration-facility.

(41) Lu, J. R.; Morroco, A.; Su, T. J.; Thomas, R. K.; Penfold, J. Adsorption of dodecyl sulfate surfactants with monovalent counterions at the air-water interface studied by neutron reflectivity and surface tension. J. Colloid Interface Sci. 1993, 158, 303-316.

(42) Li, P. X.; Li, Z. X.; Shen, H. H.; Thomas, R. K.; Penfold, J.; Lu, J. $\mathrm{R}$. Application of the Gibbs equation to the adsorption of nonionic surfactants and polymers at the air-water interface: comparison with surface excesses determined directly using neutron reflectivity. Langmuir 2013, 29, 9324-9334.

(43) Zimm, D.; Craig, V. S. J.; Kunz, W. Adsorption pattern of mixtures of trimethylammonium modified hydroxyethylcellulose and SDS at solid-liquid interfaces. Langmuir 2004, 20, 2282-2291. 